\title{
Reduced field of view under the surgical microscope due to personal protective equipment: lessons learned during the COVID-19 pandemic
}

\author{
Emrah Celtikci, MD, ${ }^{1}$ Burak Karaaslan, MD, ${ }^{1}$ Alp Özgün Börcek, MD, ${ }^{2}$ and \\ Omer Hakan Emmez, MD, PhD ${ }^{1}$ \\ 2Division of Pediatric Neurosurgery, 'Department of Neurosurgery, Gazi University Faculty of Medicine, Ankara, Turkey
}

OBJECTIVE During the coronavirus disease 2019 (COVID-19) pandemic, neurosurgeons all around the globe continue to operate in emergency cases using new self-protective measures. Personal protective equipment (PPE) use is recommended in all surgeries. The authors have experienced varying degrees of field of view (FOV) loss under the surgical microscope with different PPE. Herein, they aimed to investigate the effects of different PPE on FOV while using the surgical microscope.

METHODS Fifteen neurosurgeons and neurosurgery residents participated in this study. Three kinds of PPE (safety spectacles, blast goggles, and face shields) were tested while using a surgical microscope. FOV was measured using a $12 \times 12-\mathrm{cm}$ checkered sheet of paper on which every square had an area of $25 \mathrm{~mm}^{2}$ under the microscope. The surgical microscope was positioned perpendicular to the test paper, and the zoom was fixed. Each participant marked on the test sheet the peripheral borders of their FOV while using different PPE and without wearing any PPE. A one-way repeatedmeasures ANOVA was performed to determine if there was a significant difference in FOV values with the different PPE. RESULTS FOV was significantly different between each PPE $(F[3,42]=6339.845, p<0.0005)$. Post hoc analysis revealed a significant decrease in the FOV from the naked eye $\left(9305.33 \pm 406.1 \mathrm{~mm}^{2}\right)$ to blast goggles $(2501.91 \pm 176.5$ $\left.\mathrm{mm}^{2}\right)$ and face shields $\left(92.33 \pm 6.4 \mathrm{~mm}^{2}\right)$. There were no significant FOV changes with the safety spectacles $(9267.45 \pm$ $\left.410.5 \mathrm{~mm}^{2}\right)$.

CONCLUSIONS While operating under a surgical microscope safety spectacles provide favorable FOVs. Face shields increase the eye piece-pupil distance, which causes a severe reduction in FOV.

https://thejns.org/doi/abs/10.3171/2020.9.FOCUS20370

KEYWORDS COVID-19; neurosurgery; personal protective equipment; SARS-CoV-2

$\mathrm{T}$ HE coronavirus disease 2019 (COVID-19) pandemic is an ongoing global problem. There are recent reports recommending the delay of elective neurosurgical procedures and the use of personal protective equipment (PPE), such as N95 masks, face shields, and gowns, while operating on emergency cases. ${ }^{1}$ In Turkey, following the first confirmed COVID-19 case on March 11, 2020, the total number of cases reached 69,392 by April 15, 2020 (https://en.wikipedia. org $/$ w/index.php?title=2020_coronavirus_pandemic_ in_Turkey\&oldid=951207004). The Turkish Ministry of Health mandated the use of PPE for any surgical intervention, including neurosurgical ones. In our experience using the various kinds of PPE provided, we noticed differences in the field of view (FOV) under the surgical microscope. We aimed to investigate the effects of different PPE on the FOV while using the surgical microscope in order to recommend the most optimal choice.

\section{Methods}

A group of 15 neurosurgeons and neurosurgery residents participated in this study. Three kinds of PPE were tested: safety spectacles, blast goggles, and face shields. A surgical microscope (OPMI PENTERO 900, Carl Zeiss Meditec) was utilized in the study. FOV was measured by placing a $12 \times 12-\mathrm{cm}$ checkered sheet of paper on which

ABBREVIATIONS COVID-19 = coronavirus disease 2019; FOV = field of view; PPE = personal protective equipment.

SUBMITTED May 1, 2020. ACCEPTED September 18, 2020.

INCLUDE WHEN CITING DOI: 10.3171/2020.9.FOCUS20370. 
TABLE 1. FOV on surgical microscope with no PPE and different types of PPE

\begin{tabular}{lrrrr}
\hline \multirow{2}{*}{ Equipment } & \multicolumn{4}{c}{ FOV $\left(\mathrm{mm}^{2}\right)$} \\
\cline { 2 - 5 } Mo PPE & \multicolumn{1}{c}{ Max } & Mean & \multicolumn{1}{c}{ SD } \\
\hline Safety spectacles & 8488.42 & 9944.74 & 9305.33 & 406.1 \\
\hline Blast goggles & 2238.46 & 9851.74 & 9267.45 & 410.5 \\
\hline Face shield & 82.99 & 100.63 & 92.33 & 6.4 \\
\hline
\end{tabular}

every square had an area of $25 \mathrm{~mm}^{2}$ under the microscope. The surgical microscope was positioned perpendicular to the test paper, and the zoom was fixed. Each participant marked on the test sheet the peripheral borders of their FOV while wearing different PPE and no PPE. None of the subjects wore personal glasses during the tests. All test sheets were scanned. Measurements were performed using the ruler function of Adobe Photoshop CC 2019 software (Adobe Inc.). Statistical analyses were performed using SPSS software (version 20.0.0, IBM Corp.). A one-way repeated-measures ANOVA was performed to determine any significant difference between FOV values with the different protective equipment. A p value $<0.05$ was considered statistically significant.

\section{Results}

FOV values for the naked eye and with each of the three types of PPE are given in Table 1. Tests for homogeneity of variances demonstrated that FOV values for the naked eye were distributed normally (0.717). There were no outliers, and the cohort was normally distributed. Mauchly's test of sphericity indicated that the assumption of sphericity had been violated $\left(\chi^{2}[2]=29.333, p<0.001\right)$.
Epsilon (ع) was 0.442 and was used to correct the oneway repeated-measures ANOVA. FOV was significantly different between each PPE $(\mathrm{F}[3,42]=6339.845$, $\mathrm{p}<$ 0.0005). Post hoc analysis with a Bonferroni adjustment revealed a significant decrease in FOV from the naked eye $\left(9305.33 \pm 406.1 \mathrm{~mm}^{2}\right)$ to blast goggles $(2501.91 \pm 176.5$ $\left.\mathrm{mm}^{2}\right)$ and face shields $\left(92.33 \pm 6.4 \mathrm{~mm}^{2}\right)$. There were no significant FOV changes with safety spectacles (9267.45 \pm $410.5 \mathrm{~mm}^{2}$; Table 1, Fig. 1).

\section{Discussion}

Following the outbreak in the city of Wuhan, COVID-19 spread faster than expected. ${ }^{2,3}$ During this pandemic, neurosurgeons all around the globe were obligated to perform emergency operations including oncology cases, which require a surgical microscope, and transnasal approaches, which are associated with higher rates of infection given the relatively increased viral burden in the nasal mucosa. ${ }^{1,4-6}$ Bipolar electrocautery use during microsurgery releases electrosurgical smoke, which consists of chemicals, tissue particles, and any pathogen such as viruses. For this reason, operating teams quickly adapted to the new measures such as the new guidelines for intubation and the use of PPE. ${ }^{7}$ However, as demonstrated in our study, even as PPE protects our patients, ourselves, and our teams, it can decrease a surgeon's FOV, which can increase surgery duration and surgical complications.

Alignment of the viewer's eye and the instrument's exit pupil is essential when using an optical system. If the user is positioned far from the exit pupil and out of eye relief distance, a reduced FOV will result (https://en.wikipedia. org $/$ w/index.php?title=Eye_relief\&oldid=933756062). As an optical instrument, the surgical microscope is essential for many neurosurgical procedures. During surgery, adequate perception of the surgical area is indispensable for making appropriate and timely intraoperative decisions.

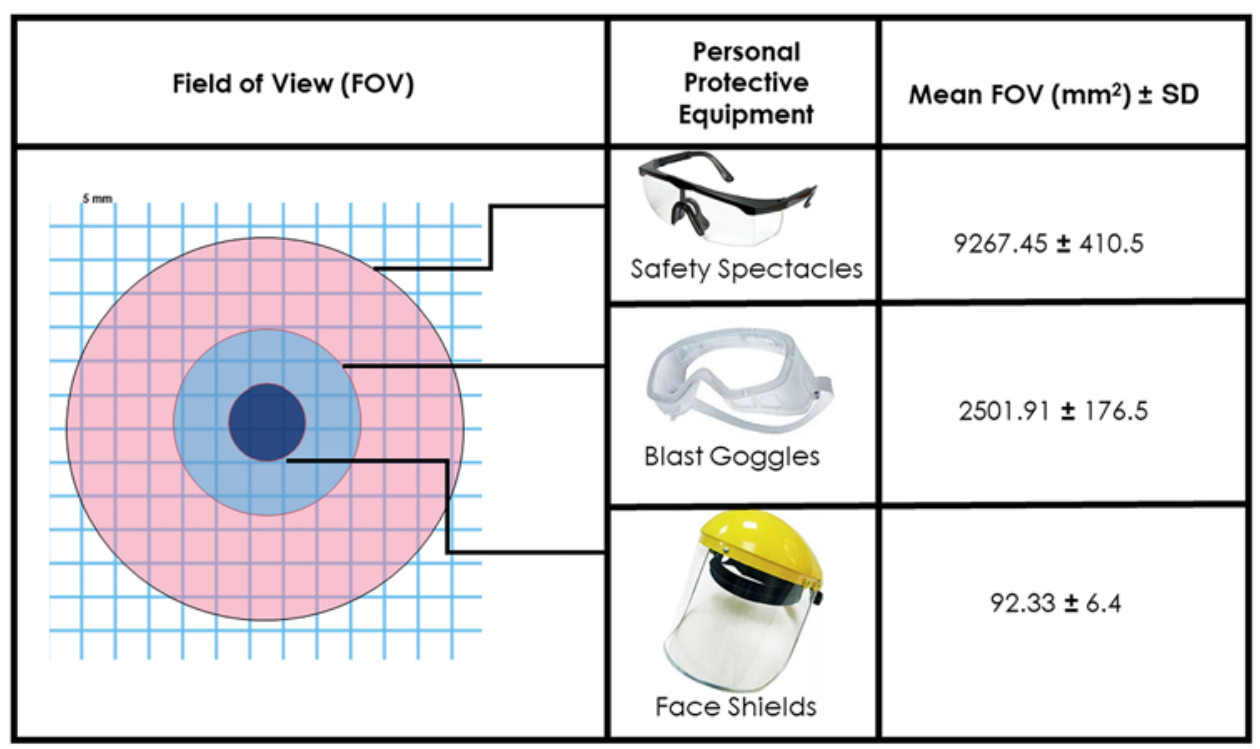

FIG. 1. Different PPE causes different FOVs on checkered paper. An increased distance from eye relief causes a decrease in the FOV. 
Alfano and Michel demonstrated that restricting the normal FOV leads to perceptual and visuomotor decrements, which will cause more errors and misperceptions. ${ }^{8}$ Additionally, they noted that subjects with restricted visual fields experience bodily discomfort.

According to our findings, PPE decreases the FOV under the surgical microscope to varying degrees depending on the extent of the distance increase from the exit pupil. Safety spectacles provide the most favorable FOV, as the distance from the exit pupil is the lowest among the PPE examined. Face shields may be convenient while using other surgical hardware such as surgical loops, a neuroendoscope, and an exoscope as well as during the craniotomy step of the cranial surgeries. Note that this study is designed for the assessment of FOV; however, new studies and quantitative measures are required to evaluate clarity of view. Any refractive material eventually changes clarity of view. Moreover, frosting of the view via steaming is another problem that we have had to deal with since the beginning of the pandemic. In the future, developing antifog PPE material is mandatory.

\section{Conclusions}

Neurosurgical practice during the COVID-19 pandemic requires new protective measures and the use of PPE. The surgical microscope is essential for most neurosurgical procedures, and an acceptable FOV is paramount for a safe surgery. PPE decreases the FOV under the surgical microscope to varying degrees depending on the extent of the distance increase from the exit pupil. Safety spectacles provide the most favorable FOV, as the distance from the exit pupil is the lowest among the PPE examined.

\section{Acknowledgments}

We thank Pinar Celtikci, MD, for assistance with proofreading and for comments that improved the manuscript.

\section{References}

1. Patel ZM, Fernandez-Miranda J, Hwang PH, et al. Letter: Precautions for endoscopic transnasal skull base surgery during the COVID-19 pandemic. Neurosurgery. 2020;87(1): E66-E67.
2. Zhu N, Zhang D, Wang W, et al. A novel coronavirus from patients with pneumonia in China, 2019. N Engl J Med. 2020;382(8):727-733.

3. Huang C, Wang Y, Li X, et al. Clinical features of patients infected with 2019 novel coronavirus in Wuhan, China. Lancet. 2020;395(10223):497-506.

4. Cenzato M, DiMeco F, Fontanella M, et al. Editorial. Neurosurgery in the storm of COVID-19: suggestions from the Lombardy region, Italy (ex malo bonum). J Neurosurg. 2020; 133(1):33-34

5. Sun Y, Mao Y. Editorial. Response to COVID-19 in Chinese neurosurgery and beyond. J Neurosurg. 2020;133(1):31-32.

6. Wellons JC III, Grant G, Krieger MD, et al. Editorial. Early lessons in the management of COVID-19 for the pediatric neurosurgical community from the leadership of the American Society of Pediatric Neurosurgeons. J Neurosurg Pediatr. 2020;26(1):1-2.

7. Orser BA. Recommendations for endotracheal intubation of COVID-19 patients. Anesth Analg. 2020;130(5):1109-1110.

8. Alfano PL, Michel GF. Restricting the field of view: perceptual and performance effects. Percept Mot Skills. 1990;70(1): $35-45$.

\section{Disclosures}

The authors report no conflict of interest concerning the materials or methods used in this study or the findings specified in this paper.

\section{Author Contributions}

Conception and design: Celtikci. Acquisition of data: Celtikci. Analysis and interpretation of data: Karaaslan. Drafting the article: Celtikci. Critically revising the article: all authors. Reviewed submitted version of manuscript: Celtikci, Emmez. Approved the final version of the manuscript on behalf of all authors: Celtikci. Statistical analysis: Karaaslan. Administrative/ technical/material support: Börcek. Study supervision: Celtikci, Emmez.

\section{Correspondence}

Emrah Celtikci: Gazi University Faculty of Medicine, Ankara, Turkey.emrahceltikci@gazi.edu.tr. 